\title{
Pause adjustment mechanism and measurement system (PAMMS)*
}

\author{
PAUL J. JENSEN \\ Communication Sciences Laboratory \\ University of Florida, Gainesville, Florida 32601 \\ KENNETH F. RUDER \\ Bureau of Child Research, University of Kansas, Lawrence, Kansas 66044 \\ and \\ WAYNE D. HARRINGTON \\ Communication Sciences Laboratory \\ University of Florida, Gainesville, Florida 32601
}

A system is described whereby silent interval duration may be studied as a parameter in the perception of speech pauses under controlled conditions. PAMMS (pause adjustment mechanism and measurement system) provides the $\mathbf{S}$ (or the E) with the unique capability of directly manipulating a pause which is then automatically measured electronically. The system includes electronic devices which provide for a specialized switching and dubbing procedure by means of which test tapes are generated.

Attention is being increasingly directed to the study of speech pauses for a variety of research purposes. Of the several types, unfilled speech pauses are of particular interest. This type is the most frequent and is readily accessible to physical identification and measurement. In the earlier literature, unfilled pauses were defined as silent intervals of some arbitrary duration (for example, Boomer, 1965; Goldman-Eisler, 1958; Hargreaves \& Starkweather, 1959). This definition provided the basis for some form of machine analysis of silences as they occurred during spontaneous speech. More recently, greater emphasis has been placed on unfilled pauses as perceptual events (Boomer \& Dittman, 1962; Martin, 1970; Martin \& Strange, 1968a, b; Ruder \& Jensen, 1971, 1972), which has necessitated greater control of experimental variables. As a result, it has become clear that, although silent interval duration is a major cue for the perception of pauses, other cues have to be taken into account as well. For example, prepausal phoneme lengthening alone (Martin, 1970; Mattingly, 1966) or in interaction with silent interval duration (Martin, 1970; Mattingly, 1966), certain postpausal effects (Mattingly, 1966), and type of phrase and clause boundary (Boomer \& Dittman, 1962; Ruder \& Jensen, 1972) have been reported to be important variables influencing the perception of pauses.

An electromechanical system

*This project was supported in part by HEW Grant NB-06459 from the National Institute of Neurological Diseases and Stroke; in part by NSF Grant GS-2337; and in part by an OCO award from the University of Florida.
(Jensen, Harrington, \& Ruder, 1971) developed for the perceptual study of speech pauses forms the basis of this report. It has the advantage of providing for direct manipulation of silent interval duration by the $S$ (or the $E$ ) in speech environments where relevant variables, such as those mentioned above, may be controlled Furthermore, it provides for immediate and repeated precise measurement of that manipulation, utilizing characteristics of the speech waveform. A methodology having these capabilities should be useful in investigating a number of variables to which pause perception is relevant, for example: grammatical boundaries (Boomer \& Dittman, 1962; Maclay \& Osgood, 1959; Martin \& Strange, 1968b; Ruder \& Jensen, 1971, 1972), syntactic processing (Martin \& Strange, 1968a, b; Ruder \& Jensen, 1971, 1972; Wilkes \& Kennedy, 1969), disambiguation of grammatical structures (Lieberman, 1967; Scholes, 1971; Ruder \& Jensen, 1972), phonetic and prosodic parameters (Martin, 1970; Mattingly, 1966), speaker pause production (Agnello, 1963; Goldman-Eisler, 1968), and individual differences in pause perception (Maclay \& Osgood, 1959; Ruder \& Jensen, 1972). The apparatus originally devised for pause manipulation in our laboratory has been reported elsewhere (Ruder, Jensen, \& Brandt, 1970). For purposes of comprehensiveness, its basic features will be reviewed. Detailed description will be given of subsequent refinements and the development of associated instrumentation which has resulted in a system applicable to pause research.

The pause adjustment mechanism and measurement system (PAMMS) consists of two major components: (1) a pause adjustment mechanism and (2) an electronic subsystem for pause measurement. In addition, it involves a specialized switching and dubbing procedure for the generation of test tapes which relates these two components. Fundamentally, the pause adjustment mechanism provides for the variable control of an interval of silence existing between two specified words of a sentence, for example the words Grant gave in the sentence "Ulysses S. Grant gave his life for his country." This control is dependent on the switching and dubbing procedure in which the segment of a sentence preceding the pause of interest is recorded on one track of a two-track tape and the following segment of the sentence is recorded on the other track. The duration of the silent interval between these two segments of the sentence may be manipulated (increased or decreased) subsequently by means of the pause adjustment mechanism. ${ }^{1}$ The resultant pause duration is measured automatically.

\section{METHOD \\ Switching and Dubbing Procedure}

To experimentally control pause (silent interval) duration between two specified words of a sentence, it is first necessary to record the indicated sentence such that the part of the sentence preceding the pause of interest $^{2}$ is recorded on Track A of a two-track tape and the following part of the sentence is recorded on Track B. The switching (from Track A to Track B) at the particular point of interest is performed electronically in order to achieve precise switching and to obviate transients audible to the $\mathrm{S}$, or sufficient to trigger the circuitry used for pause measurement.

The instrumentation and timing signals used in generation of the two-track tape are depicted in Fig. 1. The stimulus sentence is originally recorded on Track $A$ by means of a Sony TC-540 tape recorder ${ }^{3}$ with an Electro-Voice 664 microphone. This recording is subsequently made into a continuous tape loop of sufficient length to include approximately $6 \mathrm{sec}$ of silence, which provides for an interstimulus interval in repetition of the sentence during stimulus presentation. The tape loop is then played back on Channel A of the original two-channel tape recorder, and the output (Line 1) is monitored via earphones and a dual-trace storage oscilloscope. During playback of the sentence on Track A, a localizer pulse (Line 2) is recorded manually on Track B of the tape loop, by means of a pulse-delay generator in zero-delay 


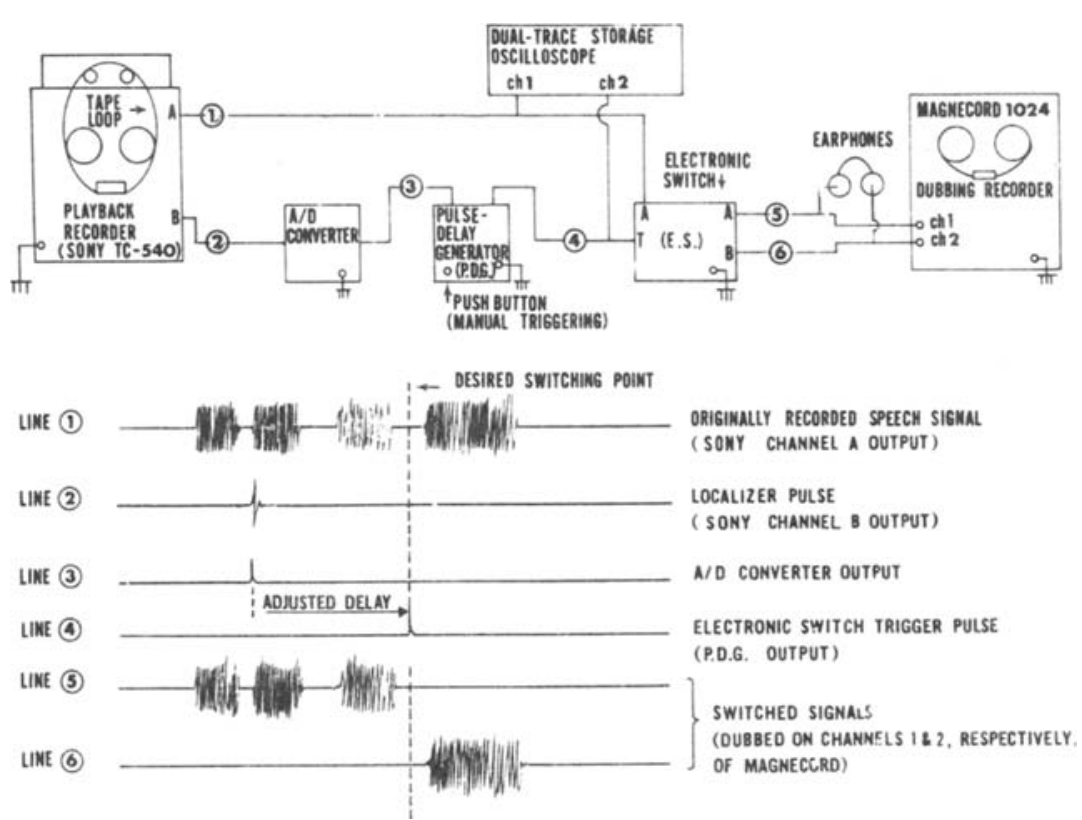

Fig. 1. Block and timing diagrams illustrating switching and dubbing procedure.

mode. This pulse is recorded somewhere between $10 \mathrm{msec}$ and 1 sec before the desired switching point. Pulse placement within this range is verified visually on Channel 2 of the oscilloscope.

The Channel A output (the entire originally recorded sentence on Track A) of the playback tape recorder is then connected to a two-channel two-track dubbing recorder (Magnecord 1024) through an electronic switch and is monitored on Channel 1 of the oscilloscope. The localizer pulse, recorded on Track $B$ after undergoing analog-to-digital conversion (Line 3), is used to trigger the pulse-delay generator, whose output, in turn, triggers an electronic switch which performs the aciual switching from Channel? to Channel 2 of the dubbing recorder.

The triggering pulse, produced b: the pulse-delay generator, is aligned with the speech signal on Track $A$ on the tape loop via manual adiustren of the pulse-delay geverator such that exact placement of the pulse withio the desired silent interva! is achimet fine 4). Accurate placement of this switching pulse is performed and verified auditorally by earphones and visually on Chimnel 2 of a duat-trade storage oscilloseope. The switched signals (Lines 5 and 6 ) are ther dubbed repeatedly isee below from the tape loop onto a reel of lape for experimental use.

Figures 2 and 3 show oscilloscope tracings of an utterance that was switched from one track of a tape to another using this procedure. The desired point of switching. Figure 3 is a photograph of oscilloscope tracings showing a delayed sweep of the portion of the utterance where the switching is to be performed (Trace 1), the electronic switch triggering pulse (Trace 2), and the switched signals (Traces 3 and 4). This photograph shows clearly that the switching procedure did not disturb the temporal relations between the two words (Grant gave), nor is there any visible evidence of a switching transient rising above the baseline noise.

Once the speech signal input to the dubbing recorder is being switched from Channel 1 to Channel 2 at the desired interval, the tape loop on the payback recorder is allowed to cycle continuously and the switched signal is dubbed repeatedly on a single reel of tape such that the portion of the sentence preceding the switching point is recorded on Track $A$ and the remainder of the sentence is recorded on Track B. The switched stimulus sentence is dubbed repeatedly on a single reel of tape in order to provide the Ss with as many repetitions of the sentence as necessary in making the pause adjustments required in the experimental task.

As indicated in Fig. 1, the instrumentation required for this switching and dubbing procedure consists of two stereo tape recorders (each channel of each tape recorder must be capable of recording and/or playing back separately), a dual-trace storage oscilloscope, a pulse-delay

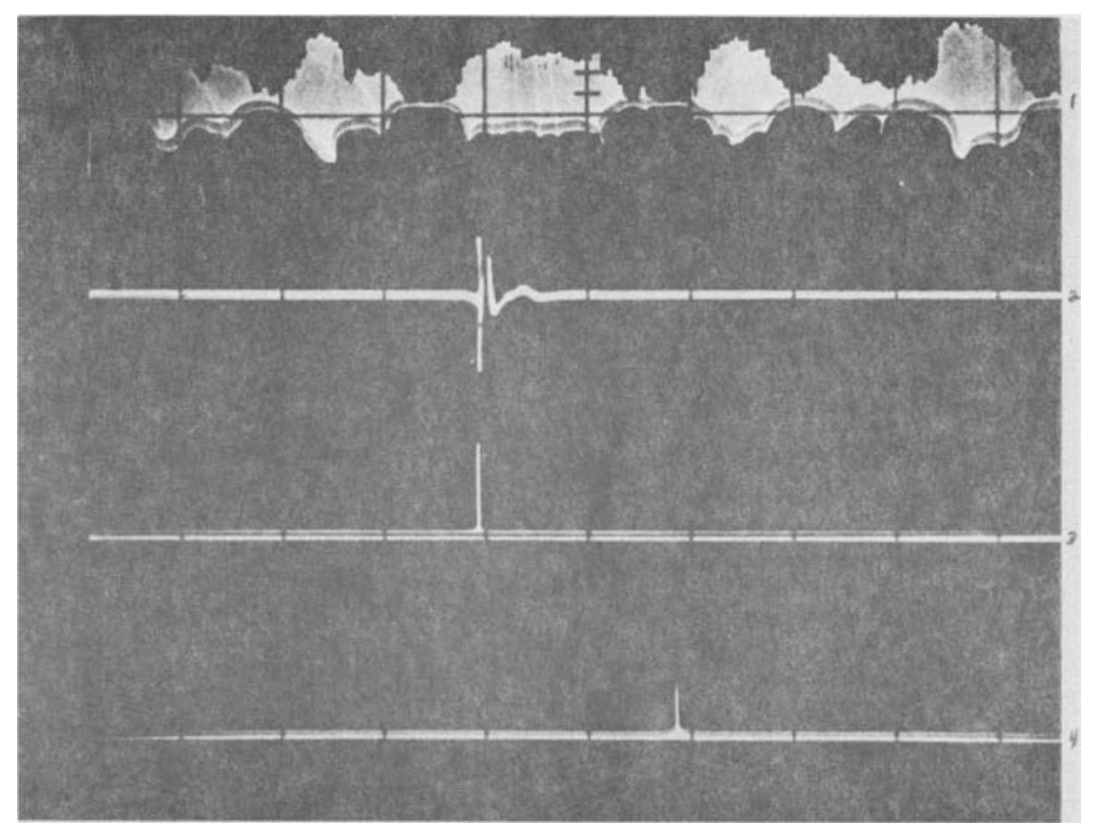

Fig. 2. Oscilloscope tracings of actual utterance and switching signals. 
generator (P.D.G.) and an electronic switch (E.S.). To reduce the expense of instrumenting, ${ }^{5}$ the pulse-delay generator and electronic switch were constructed from inexpensive microelectronic hardware. These components were designed specifically to meet the needs of the PAMMS switching procedure. While not as versatile as their commercial counterparts, ${ }^{6}$ they do provide satisfactory electronic performance.

The pulse-delay generator constructed for PAMMS provides for pulses of zero delay (for recording the localizer pulse on Track B of the original tape loop), as well as pulses of variable delay (for triggering the electronic switch at the desired point of switching from Track $A$ to Track B). The selection of either of these modes is available through a delay/zero-delay switch on the device. Lines 3 and 4 of Fig. 1 illustrate the operation of this functional block (in this case operating in the delay mode). A pulse serves as input to the device; an adjustable number of milliseconds later, the device provides an output pulse. By means of this particular pulse-delay generator, pulses can be delayed from a minimum of $4 \mathrm{msec}$ to a maximum of $1.1 \mathrm{sec}$.

The functional operation of the electronic switch is also shown by Fig. 1. This device has two inputs. One of these is the signal to be switched; the other input is the control signal (pulse) which activates the switching function. These are diagrammatically shown in Fig. 1 as Inputs $A$ and $T$, respectively. The outputs of the electronic switch are denoted as $\mathrm{A}$ and $B$ (Lines 5 and 6 ). The input signal (Line 1) appears on output Channel A (Line 5) prior to switch triggering, i.e., when the switch is in its "rest" state. When a triggering pulse appears on the $\mathrm{T}$ input (Line 4), Channel A turns $O F F$ and Channel B turns ON. Channel $B$ remains $O N$ for an adjustable period of time, whereupon the switch reverts to its previous state (A-ON, B-OFF). The switch, therefore, contains its own time base, which is adjustable for $\mathrm{ON}$ times ranging from $35 \mathrm{msec}$ to $30 \mathrm{sec}$. The switching of signals by this functional block is shown by the timing of signals on Lines $1,4,5$, and 6 of Fig. 1 .

The pulse-delay generator and electronic switch provide precise switching of speech from one tape recorder channel to another. Repeated switching of the same speech signal has d emonstrated that this instrumentation is extremely reliable (within microseconds from one time to the next) and accurate. The electronic switch, furthermore, produces no audible transient in making the switch, nor is the transient

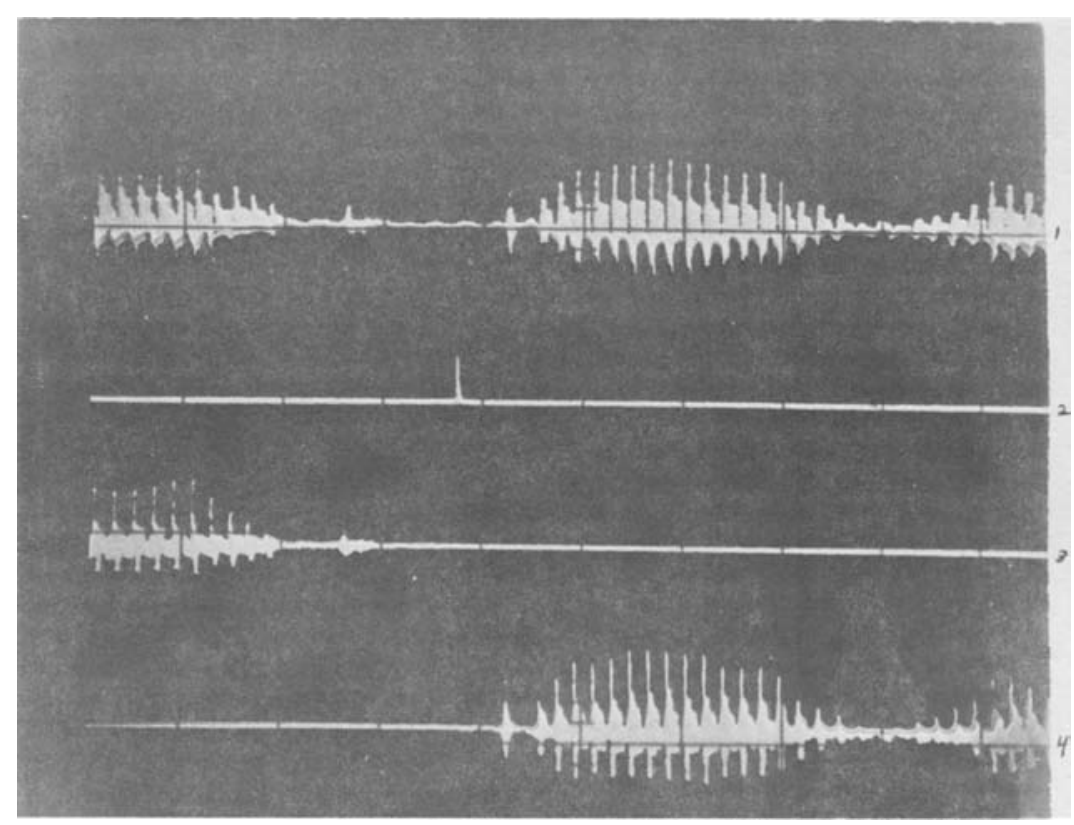

Fig. 3. Oscilloscope tracings (from top to bottom) showing unaltered temporal relations between unswitched (Trace 1) and switched speed signal (Traces 3 and 4 ); Trace 2 shows the switching pulse.

sufficient to trigger the speech pause measurement circuit to be described later.

Pause Adjustment Mechanism

The test tapes, prepared by means of the switching and dubbing procedure, are then used in conjunction with the pause adjustment mechanism. The basic principle of operation and construction of the PAMMS pause adjustment mechanism is like that reported by Ruder, Jensen, \& Brandt (1970). Fundamentally, the mechanism provides for variable control of an interval of silence between the word terminating the Track $A$ signal and that initiating the Track B signal of the two-track test tape. Pause duration is accomplished through delaying to a greater or less degree, the playback of the Track B signal relative to the signal on Track $A$. To provide for such a variable delay, a tape guide attached to a worm gear assembly, as shown in Fig. 4, was mounted between the fixed Track A and B playback heads. Since the tape guide is attached to the worm gear, its position, relative to the horizontal plane of the playback heads, can be moved up and down the worm gear, thereby increasing or decreasing the effective distance between the playback heads. Pause duration (time interval between the playback of the Track $A$ and $B$ signals) between the experimental word sequences can be increased, then, by moving the tape guide away from the playback heads or decreased by moving the tape guide toward the playback heads, since this movable guide alters the time of onset of the Track B signal relative to that on Track A.

A refinement of the PAMMS pause adjustment mechanism is that the worm gear is now driven by a variable speed "fast" for gross adjustment and "slow" for fine adjustment) reversible motor. The position of the movable tape guide can thus be controlled by the $\mathbf{S}$ who operates the motor by means of a remote control unit or by the $E$ through a master control unit (see Fig. 4). The control units are also equipped with signal lights to indicate when the $S$ is to start the adjustment (signal controlled by the E) and when he has completed the adjustment (S signals E). Signal lights, activated by microswitches, also indicate on both control units when the upper or lower limit of the pause adjustment mechanism has been reached. Use of the motor drive and remote control assembly has several major advantages over the earlier hand crank system, namely: (1) Pause adjustment can be accomplished by the $\mathrm{S}$ more rapidly and effectively using the multispeed adjustment, especially when he has to make a large adjustment, as for hesitation pauses, and (2) the equipment can be more effectively isolated from the $S$, thus reducing the auditory and visual distractions present with the previous apparatus. 


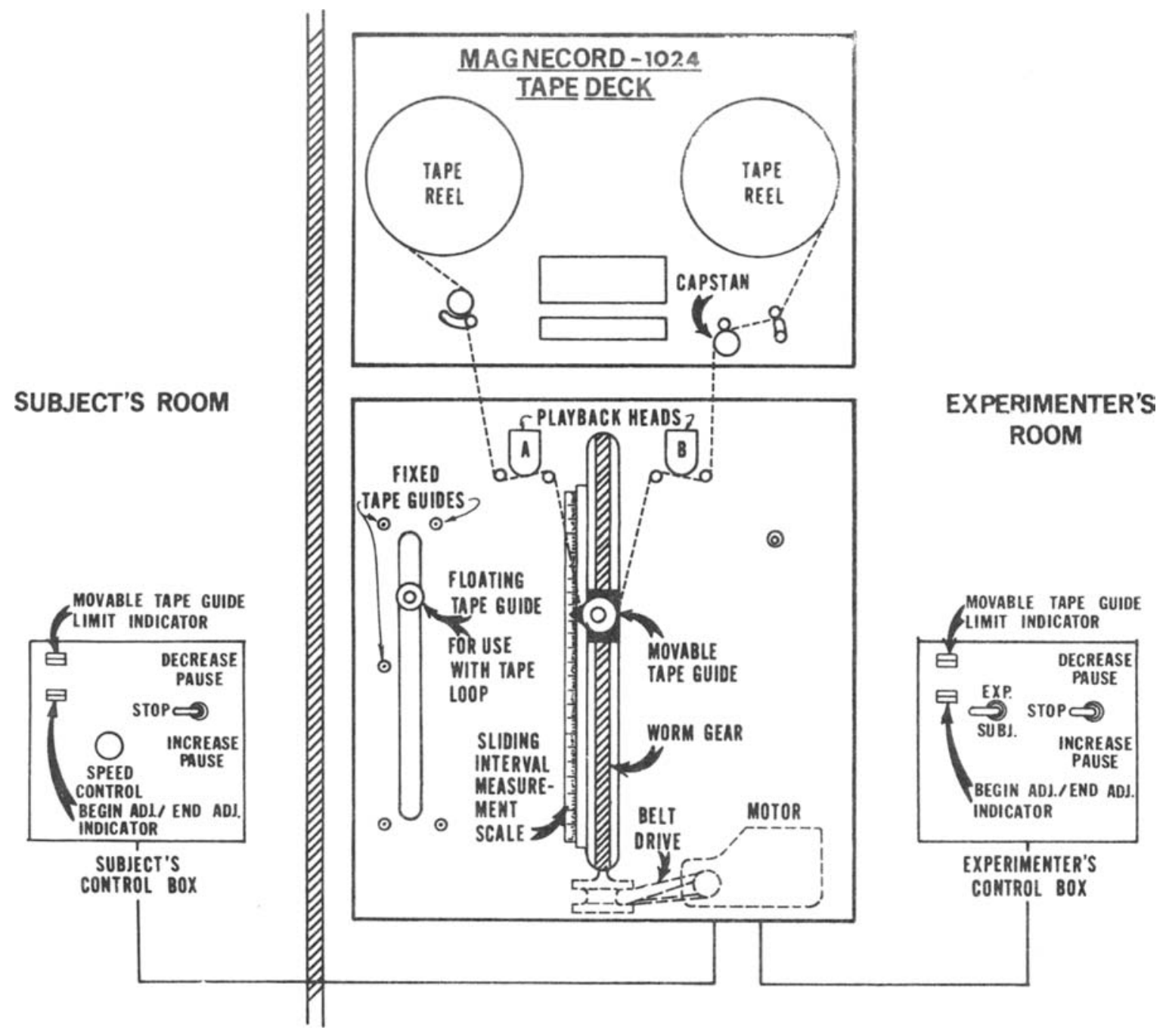

Fig. 4. Block diagram of pause adjustment mechanism and control units.

A second refinement concerns the record mode. Because of the physical separation of the PAMMS Track A and $B$ playback heads, it is impossible to achieve a zero pause adjustment without a manipulation of the temporal relations between the Track $A$ and $B$ signals. To provide the capability of adjusting the pause duration between the Track $A$ and $B$ signals to a value less than that present in the original recording, or to go to absolute zero duration or word overlap if desired, it is necessary to record the tapes to be presented to the Ss. This is done such that the Track $B$ signal is physically advanced on the tape with respect to the Track $A$ signal by a duration greater than the time represented by the separation of the PAMMS playback heads plus the silent interval duration of the original recording. In the earlier approach, a manual dubbing procedure was employed which provided for such advancement of the Track B signal. The PAMMS modifcation, however, allows one to perform this operation automatically without the necessity of redubbing as in the previous method.

The improvement is due to a modification of the tape transport head assembly. In brief, this involved interchanging the Channel 2 and Channel 1 record heads ${ }^{7}$ and installing a roller-bearing type of tape guide approximately $6 \frac{1 / 2}{\mathrm{in}}$. directly above these record heads. As a result, the Channel 2 signal (on Track B) is recorded earlier than the Channel 1 signal (on Track A) by the amount of time corresponding to the new tape distance separating the recording heads. This distance is approximately 15 in. By using this modified recording setup for the dubbing recorder (see Fig. 1) in the switching and dubbing procedure previously described, the
Track B signal is recorded approximately $2 \mathrm{sec}$ (71/2 ips) ahead of the Track A signal. This corresponds, then, to the maximum amount of temporal overlap between the Track B and Track A signals which the PAMMS procedure provides.

The maximum tape distance between the two playback heads is approximately 43.5 in., which corresponds to $5.8 \mathrm{sec}$ at $7 \frac{1}{2}$ ips. This amount, less approximately 2 sec of Track A and B overlap, corresponds to the maximum pause duration of which PAMMS is capable (in its present configuration), that is, approximately 3,800 msec. Thus, PAMMS has the capability of overlapping two sentence segments by as much as $2 \mathrm{sec}$ and of separating these segments by as much as approximately 4 sec.

The pause adjustment mechanism of PAMMS is coupled to a Magnecord 1024 tape deck and stereo amplifier 
assembly driving a pair of Sharpe HA-10 earphones with a monaural Y-cord input so that Track $A$ and Track B signals are received in both ears. ${ }^{8}$ To keep the distance between the playback head stacks and the capstan drive to a minimum, the pause adjustment mechanism was mounted directly below the tape transport. The amplifier assembly was thus mounted above the tape transport rather than below, as is generally the case.

The effect of the pause adjustment mechanism on the tape-recorded signal has been examined. Signal-to-noise ratio, wow and flutter, and frequency response were all within the factory specifications at both minimum and maximum delays possible. Thus, no appreciable distortions are introduced in the tape-recorder system through the addition of the delay device.

Pause IMeasurement System

The electronic technique used in measurement of the speech pause (more specifically, the adjusted silent interval) relies on analog-to-digital information necessary to actuate an electronic counter which performs the time computation function. This information can be transmitted to a recorder that provides a printout in digital form.

The most general functional description of the measurement system, plus a diagram portraying the waveform timing relations, is presented in Fig. 5. The prerecorded stimulus sentence, after being adjusted for the desired pause duration by the $S$, is played on Channels 1 and 2 of the input tape recorder 9 into the measurement circuit shown within the dashed lines. The general appearance of these speech waveforms is shown as the information appearing on Lines 1 and 2 of the waveform timing diagram. The pause (silent interval) to be measured is denoted as P. The purpose of the system is to first transmit a start-count pulse to the electronic counter at the exact time corresponding to the termination of speech energy on Line 1 , as shown in the waveform diagram. This is accomplished by the electronics within the functional block labeled Pulse Delay. The start-count pulse appears on Line 3, as indicated, and is labeled ST. Positioning of this pulse for a given stimulus sentence is performed manually, as described below.

After the start-count pulse has been transmitted, the electronic counter (adjusted to time interval measurement mode) begins the count of elapsed time and continues to count until it receives a stop-count pulse (labeled STP on the waveform timing diagram). This pulse is generated by the electronics within the block

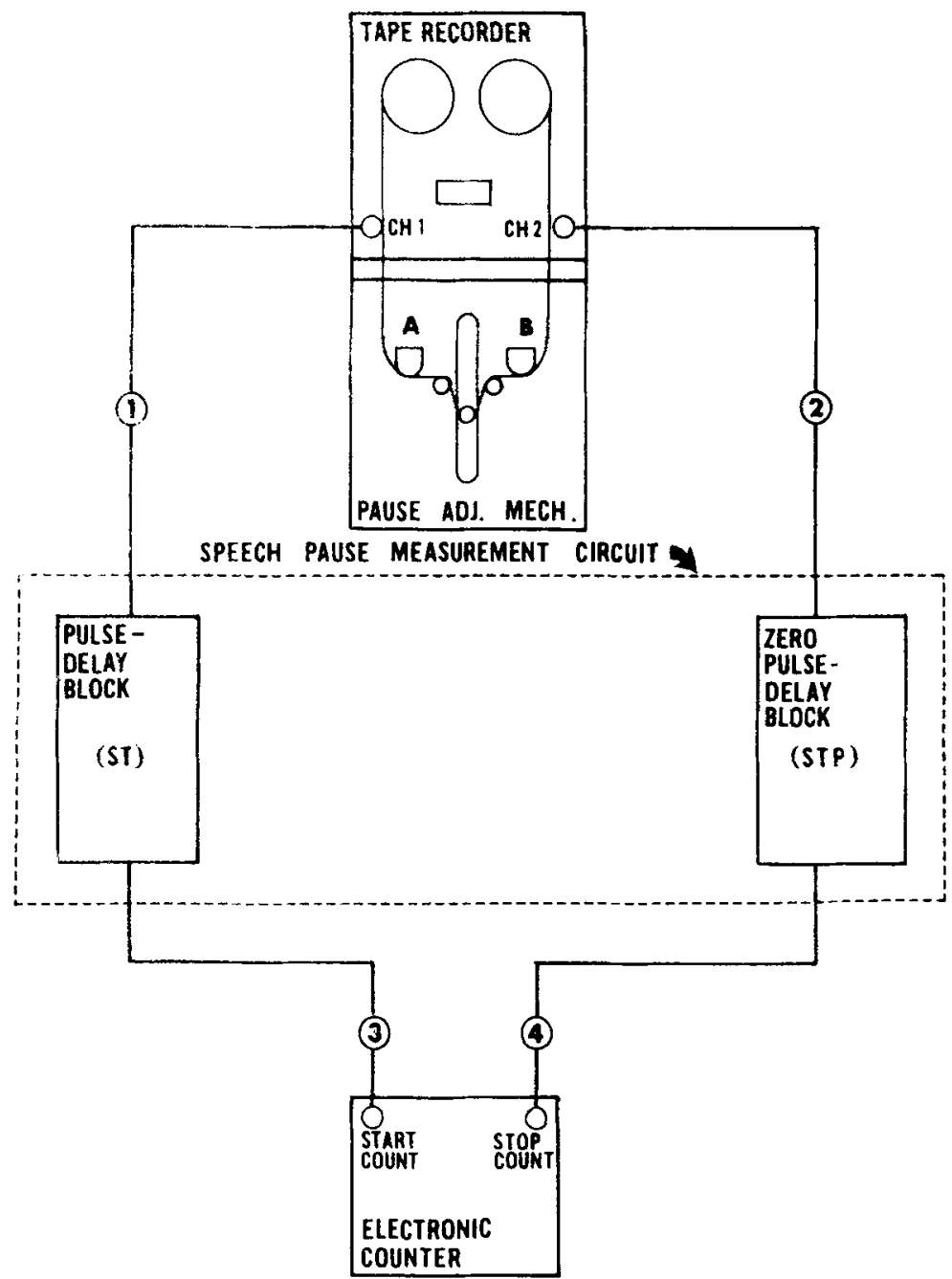

WAVEFORM TIMING DIAGRAM

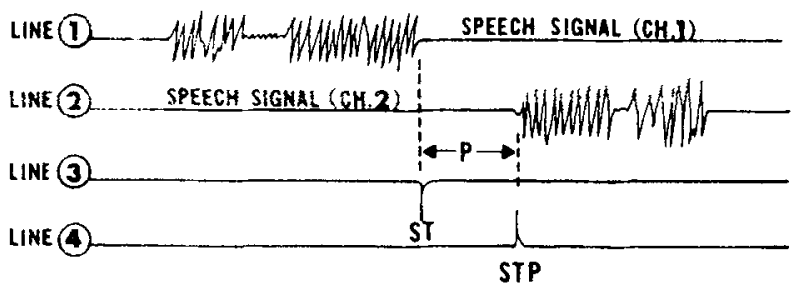

Fig. 5. Functional description of approach used in pause measurement consisting of schematized signal input and output devices, measurement circuit, and waveform timing relations.

labeled Zero Pulse-Delay. The stop-count pulse is generated by this block immediately upon encountering speed energy, as shown by Line 2 . The count of elapsed time in milliseconds that has occurred during the interval between these two pulses (Lines 3 and 4) represents the duration of the silent interval being measured and is displayed by the counter. This count may be immediately repeated for reliability purposes. In addition, it may be corroborated by visual measurement of the adjusted interval from the oscilloscope face and by a check of the interval measurement scale (Fig. 4) of the pause adjustment mechanism.

Figure 6 depicts the electronic subblocks 10 which comprise each of the more general blocks illustrated in the previous figure. As before, it also displays some of the internal waveforms in diagram form which are generated by these subblocks. The components of the pulse-delay block 
PULSE - DELAY BLOCK

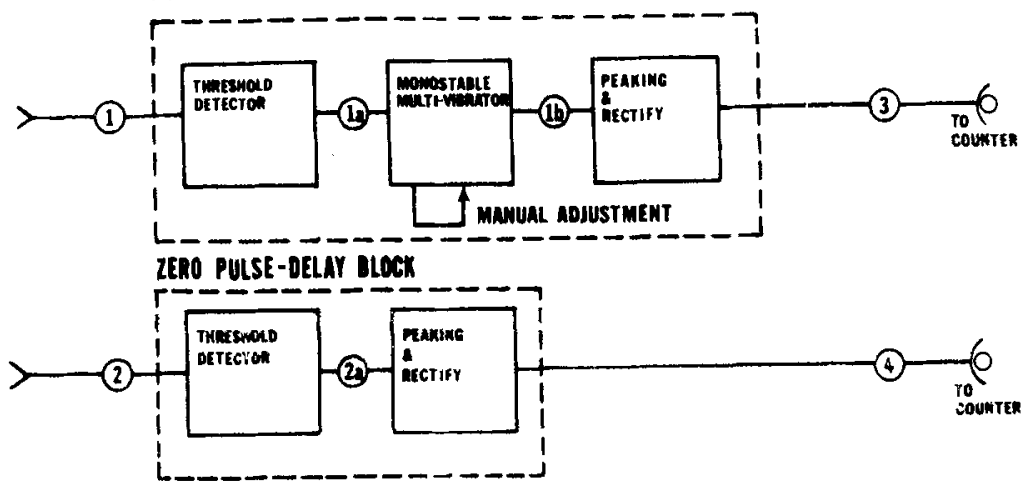

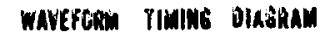
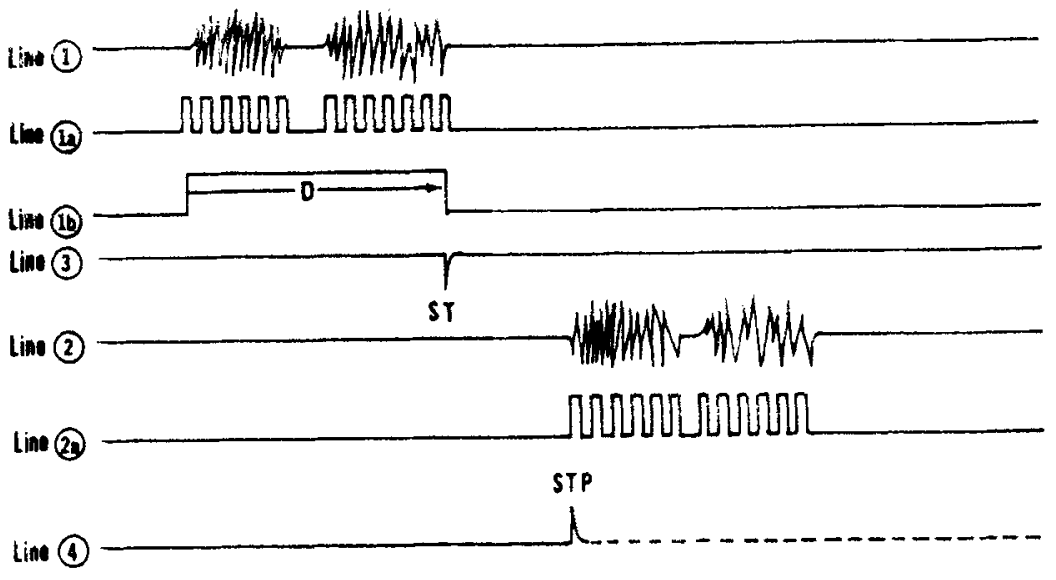

Fig. 6. Speech pause measurement circuit: subblock functional description and waveform timing relations.

are shown within the upper dashed lines. The threshold detector performs $A / D$ conversion on the incoming speech, as indicated by Lines 1 and $1 \mathrm{a}$.

The leading edge of the first of these pulses triggers a monostable multivibrator into its metastable state, as shown by Line $1 \mathrm{~b}$. The duration of this state, labeled $D$, is manually adjusted so that the state terminates at the exact time that the speech signal on Line 1 terminates. This manual adjustment initially involves the selection of one of four pulse-delay ranges by means of a four-position switch. These ranges are as follows: $180-870,260-1,300,350-1,740$, and $700-3,500$ msec. An appropriate delay selection is one that corresponds to the approximate duration of the switched sentence segment on Channel 1 as measured on the oscilloscope screen.

The state changes of the monostable multivibrator, which has been adjusted for an appropriate delay range, are differentiated by a peaking network and rectified such that only peaking pulses of negative polarity are transmitted. The resultant output is used as the start-count pulse, ST, as manual method, however, has particular merit in providing the capability of very precise start-count pulse placement.

The zero pulse-delay subblocks of Fig. 6 are the same as the pulse-delay subblocks except that the monostable multivibrator is deleted. The internal waveforms associated with the zero pulse-delay block are shown as Lines $2,2 a$, and 4 of Fig. 6 . These waveforms are generated in the identical manner as the waveforms in the pulse-delay block. Figure 9 shows a photograph of these various timing relations in the actual measurement of an adjusted pause. In this instance, the pause measured was $396 \mathrm{msec}$.

\section{RELIABILITY}

Two types of reliability assessments were made of PAMMS. Both involved six stimulus sentences which had been prepared using the PAMMS instrumentation and procedures described previously in this article. The word pairs between which the switching and dubbing, pause adjustment, and pause measurement procedures took place are underlined in the sentences listed in Table 1. Notice that in each instance the first word of the pair ends with a stop-plosive consonant and the second word of the pair begins likewise with a stop-plosive consonant. This selection was made because of the particular ease of start-count placement in this phonetic environment. However, as indicated earlier, ${ }^{2}$ the PAMMS technique can be employed with any two words of a sentence that are adjacent, regardless of their phonetic termination and initiation, 11 so long as there exists between them some brief interval of silence that may be manipulated.

Both methods of reliability assessment involved playing the stimulus sentences through PAMMS from the beginning to the end of each tape reel. Each 7-in. reel contained approximately 200 repetitions of a stimulus sentence. The entire series of sentences was played at the following approximate settings of the pause adjustment mechanism: $5,25,100$, $250,500, \quad 1,500,3,000$, and 3,700 msec. On the first and every succeeding 10 th sentence repetition, the silent interval duration at the point of pause manipulation was visually measured on the storage oscilloscope screen and compared with the reading displayed by the counter. There was agreement in all instances. 12

Table 2 lists the mean deviations (in milliseconds ) of repeated measurement for each pause setting covering the range of the pause adjustment mechanism for each of the stimulus sentences. In all, 9,600 data points are 
represented by the 48 mean deviations of Table 2, that is 200 sentence repetitions at each of eight pause settings for each of six sentences.

It is clear from Table 2 that PAMMS produces highly consistent data. Since the largest mean deviation is $2.0 \mathrm{msec}$ and the smallest is $0.5 \mathrm{msec}$, the general statement may be made that the average error of measurement is $1.5 \mathrm{msec}$ or less, regardless of pause setting, stimulus sentence, or where the sentence is located on the test tape. It should be understood that the slight variation that does exist in the reliability data represents not just the variation due to the various components of the pause adjustment mechanism and the measurement system, including the electronic counter, but also that due to the switching and dubbing procedure, as well as variation in tape recorder speed and signal amplitude during recording and playback. Thus, the data contained in Table 2 represent the variability present in the entire PAMMS approach.

We recognize, of course, that error as small as that shown in the table is perceptually insignificant. Reliablity as

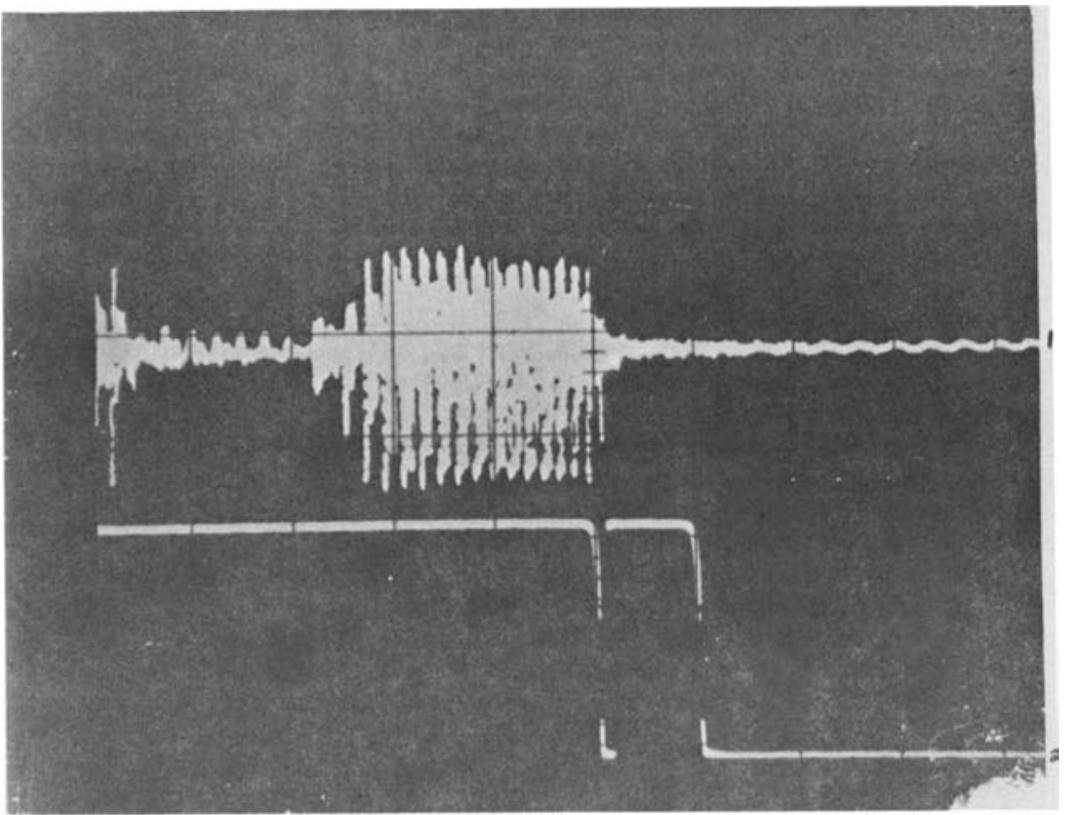

Fig. 7. Oscilloscope tracings showing appearance of double pulse during inappropriate positioning of start-count placement at terminal edge of speech waveform.

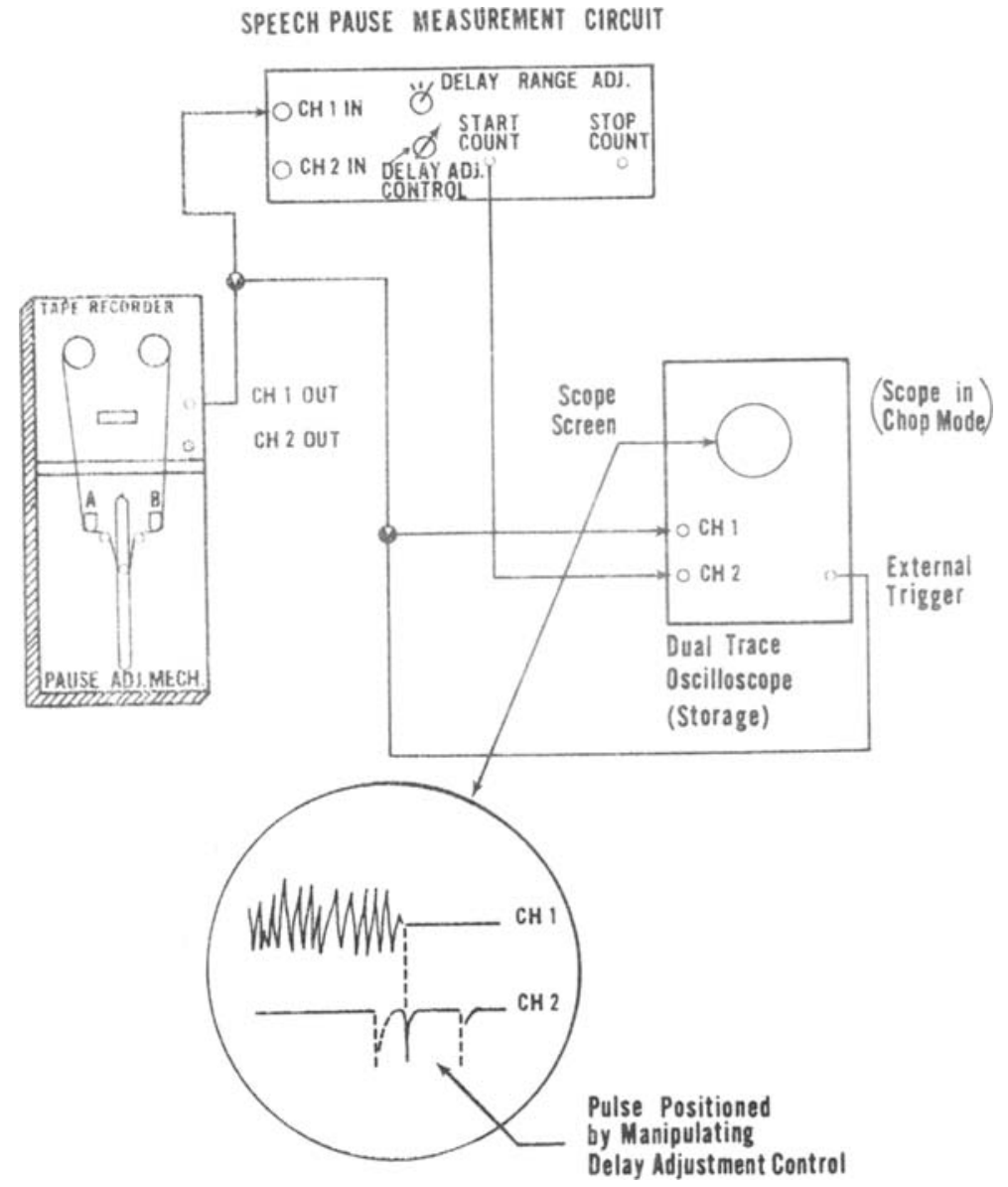

Fig. 8. Block diagram of instrumentation to illustrate start-count pulse placement. great as this, however, gives reassurance about the system for research purposes and may be crucial for certain types of investigations employing the PAMMS methodology. More precise and reliable data could be obtained if FM instrumentation tape recorders replaced the AM tape recorders presently being used or if computer-assisted technology was employed. Either approach, of course, involves substantially higher costs.

\section{ILLUSTRATIVE APPLICATIONS}

Psycholinguistic studies (Ruder \& Jensen, 1971, 1972), conducted with an earlier version of the pause adjustment mechanism, exemplify one type of research application of the system. A major question under investigation was whether pauses during speech encoding serve to reflect the syntactic structure of the sentence for purposes of decoding. Listeners were asked to perform judgments of what constituted a minimally perceived pause, an optimal fluent pause, and a minimal hesitation pause as the syntactic relation of an experimental word pair was varied systematically in complexity from sentence to sentence. In general, for each of the pause types, there were nonsignificant differences between pause durations and level of syntactic complexity. These findings supported the proposal by Martin and Strange $(1968 \mathrm{a}, \mathrm{b})$ that pauses reflect the organizational processes of the speaker, but are redundant as cues in this regard for the listener. The 


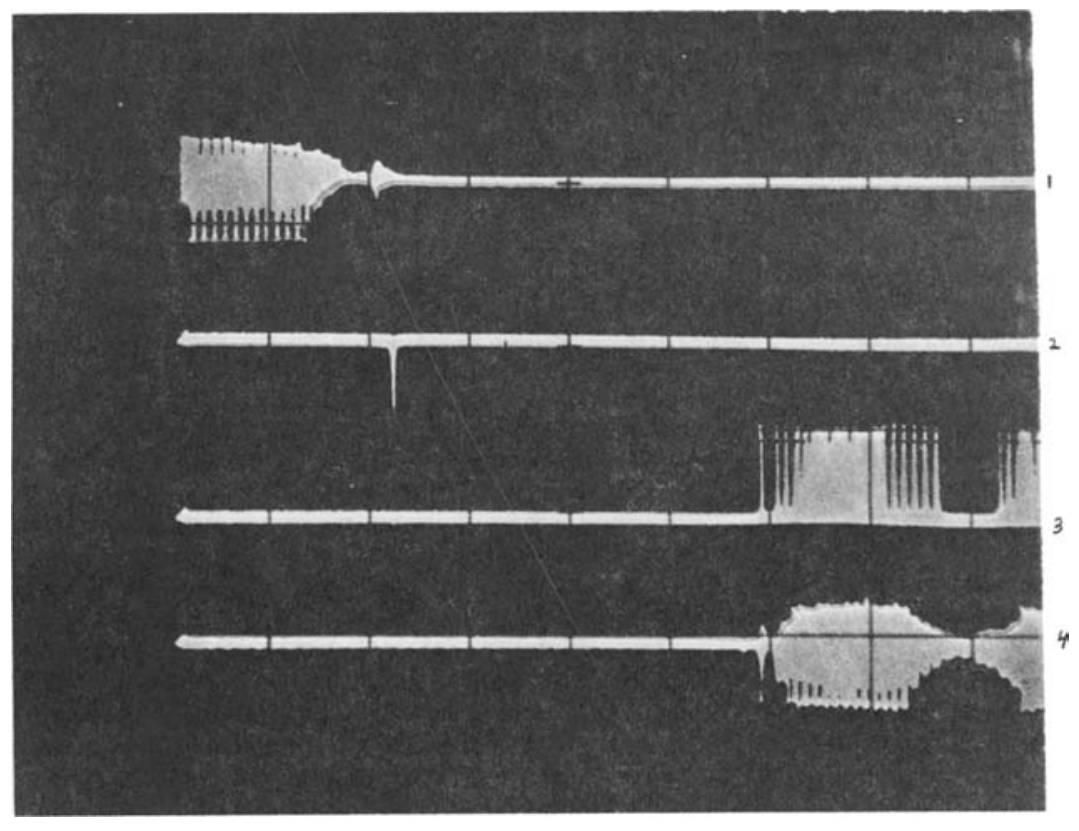

Fig. 9. Oscilloscope tracings showing pause measurement timing relations.

Table 1

Stimulus Sentences on Which Reliability of PAMMS Was Assessed

The embarrassed cop tore up the
ticket.
2 The injured dog took refuge in the
barn.
3 The US Hope gives medical care to
the needy.
4 The leak discharged oil into the lake.
5 Ulysses S. Grant gave his life for
his country.
The duck became separated from the
flock.

described methodology would be very useful in establishing this conception more firmly by employing additional syntactic material so as to generalize these findings.

Of interest also was the question of how reliable listeners are in pause perception. Repeated judgments of the pause types at the various levels of syntactic complexity were highly consistent. There were substantial differences between listeners, however, which parallel the results found in studies of pause production in spontaneous speech (Goldman-Eisler, 1968). These results suggest the need to study whether or not speakers perceive pauses with reference to their production of pauses. Information of this kind, of course, has relevance to current theories of speech perception. 3 The PAMMS methodology lends itself readily to such investigations.

A logical extension of this theoretical orientation and methodology is to the problem of of a 7-in. tape reel.

tApproximate phonetic elements of speech signals. Of particular relevance in this regard are studies of perceptual segmentation (Huggins, 1972). Although the pause adjustment mechanism and measurement system was specifically developed for the perceptual study of speech pause phenomena, it is evident that the instrumentation and procedures which it comprises may be utilized in a variety of applications for speech and language research.

\section{REFERENCES}

AGNELLO, J. A study of intra- and inter-phrasal pauses and their relationship to rate of speech. Unpublished doctoral dissertation, Obio State University, 1963.

BOOMER, D. S. Hesitation and grammatical encoding. Language \& Speech, 1965, 8, 148-158.

BOOMER D. S., DITTMAN, A. T. Hesitation pauses and juncture pauses in speech. Language \& Speech, 1962, 5. 215-220.

FEW I $R$ \& LINGWALL, J, B, A further analysis of fluency within stuttered speech. Journal of Speech \& Hearing Research 1972, 15, 356-363.

GOLDMAN-EISLER, F. Speech production and the predictability of words in context. Quarterly Journal of Experimental Psychology, 1958, 10. 96-106.

GOLDMAN-EISLER, F. Psycholinguistics: Experiments in spontaneous speech. New York: A cademic Press, 1968.

HALLE, M., \& STEVENS, K. N. Speech recognition: A model and a program for research. In J. A. Fodor and J. J. Katz. (Eds.), The structure of language. Englewood Cliffs, New Jersey: Prentice-Hall, 1964. Pp. 604-612.

H R G R E A E S W. A., \& STARKWEATHER, J. Collection of temporal data with the duration tabulator. Journal of the Experimental Analysis of Behavior, 1959, 2, $179-183$

HUGGINS, A. W. F. An electronic tape-splicer and synchronizer. Quarteriy Journal of Experimental Psychology, $1970,22,337-340$

HUGGINS, A. W. F. On the perception of temporal phenomena in speech. Journal of the Acoustical Society of America, $1972,51,1279-1290$.

IENSEN, P. J., HARRINGTON, W. D., \& RUDER; K. F. Pause adjustment mechanism andd measurement system (PAMMS). Quarterly Progress Report, $1971,9: 1,33-45$. Communication Sciences Laboratory, University of Florida. (This is a preliminary and brief description of the apparatus and

Table 2

Mean Deviations (in Milliseconds) of Repeated Measurement for Eight Pause Settings (in Milliseconds) for Each of Six Stimulus Sentences*

\begin{tabular}{rrrrrrr}
\hline $\begin{array}{c}\text { Sentences } \\
\text { Pause }\end{array}$ & 1 & 2 & 3 & 5 & 5 \\
\hline Settingst & & & Mean & Deviations (Range = 1.5) \\
\hline 3700 & 1.4 & 1.1 & 1.4 & 1.3 & 1.4 & 0.8 \\
3000 & 1.9 & 0.9 & 1.6 & 1.4 & 1.9 & 0.9 \\
1500 & 1.6 & 0.9 & 1.3 & 1.5 & 1.3 & 0.6 \\
500 & 1.6 & 0.9 & 1.5 & 1.4 & 1.3 & 0.7 \\
250 & 1.7 & 1.0 & 1.6 & 1.2 & 1.5 & 0.9 \\
100 & 1.6 & 0.8 & 2.0 & 1.4 & 1.3 & 1.0 \\
25 & 1.5 & 0.9 & 1.3 & 1.0 & 1.2 & 0.6 \\
5 & 1.3 & 1.1 & 1.6 & 1.3 & 1.1 & 0.5 \\
\hline
\end{tabular}

*Each mean deviation represents 200 sentence repetitions from the beginning to the end 
techniques reported in detail in the present article.)

JOHNSON, W., \& ASSOCIATES. The onse of stuttering: Research findings and implications. Minneapolis: University of Minnesota Press, 1959. P. 262.

LIEBERMAN, P. Intonation, perception and language. Cambridge, Massachusetts M.I.T. Press, 1967.

LOVE, L., \& JEFFRESS, L. Identification of brief pauses in the fluent speech of stutterers and nonstutterers. Journal of Speech \& Hearing Research, 1971, 14 , 229-240.

MACLAY, H., \& OSGOOD, C. E. Hesitation phenomena in spontaneous English speech. Word, 1959, 15, 19-44.

MARTIN, J. G. On judging pauses in spontaneous speech. Journal of Verbal Learning \& Verbal Behavior, 1970, 9, 75-78.

MARTIN, J. G., \& STRANGE, W. Determinants of hesitations in spontaneous speech. Journal of Experimental Psychology, 1968a, 76 474-479.

MARTIN, J, G., \& STRANGE, W. The perception of hesitation in spontaneous speech. Perception \& Psychophysics, $1968 \mathrm{~b}, 3,427-438$.

MATTINGLY, I, G. Synthesis by rule of prosodic feat:dres. Language \& Speech, $1966,9,1-13$

RUDER, K. F., \& JENSEN, P. J. Speech pause duration as a function of syntactic junctures. In E. Foulke (Ed.), Proceedings of the Second Louisville Conference on $R$ ate andior Frequency-Controlled Speech. Center for Rate-Controlled
Recordings, University of Louisville, 1971. Pp. 119-133.

RUDER, K. F., \& JENSEN, P. J. Fluent and hesitation pauses as a function of syntactic complexity. Journal of Speech \& Hearing Research, 1972, 15, 49-60.

RUDER, K. F., JENSEN, P. J., \& BRANDT J. F. An apparatus and procedure for the perceptual study of speech pauses. Journal of the Experimental Analysis of Behavior, 1970, 14, 287-289.

RUPF, J. A., ROWSON, S. V., \& RUDER K. F. A method for the precise switching of speech. Behavior Research Methods \& Instrumentation, 1972, 4, 11-12.

SCHOLES, R. J. On the spoken disambiguation of superficially ambiguous sentences. Language \& Speech, 1971, 14, 1-11.

WILKES, A., \& KENNEDY, R. Relationship between pausing and retrieval latency in sentences of varying grammatical form. Journal of Experimental Psychology, $1969,79,241-245$

\section{NOTES}

1. A somewhat similar method for adjustment of segmental phoneme duration has been reported by Huggins (1970). The present technique has this same potential, but by means of encoded electronic pulses rather than photoelectric sensing.

2. Actually, any interval of silence existing between any two adjacent words of a sentence.

3. This is a two-channel four-track machine. Our technique employs only two tracks ( $A$ and $B$ ).

4. In this instance, the photograph has been touched up in order to make the pulse clearly evident

5. Schematics for any of the electronic instrumentation described in this paper may be obtained from the senior author.

6. If commercial components are used for

this switching procedure, the instrumentation described by Rupf, Rowson, \& Ruder (1972) is more appropriate than that depicted in Fig. 1.

7. It should be remembered that the reproduce heads for Channel 1 and Channel 2 of PAMMS are mounted on the pause adjustment mechanism, as shown in Fig. 4.

8. An important aspect of the PAMMS procedure, incidentally, is balancing of Channel 1 and Channel 2 for recording and playback levels. A special calibration procedure using a test tone from an oscillator is employed for this purpose.

9. Modified to incorporate Playback Heads $A$ and $B$ of the pause adjustment mechanism.

10. An overall circuit schematic which presents all of the electronic requirements for these subblocks is available from the senior author.

11. Theoretically, at least. Our experience thus far with PAMMS has involved only the stop-phoneme environment.

12. Within the reading parallax of the oscilloscope time base grid.

13. Particularly the analysis-by-synthesis model of speech perception described by Halle \& Stevens (1964). 